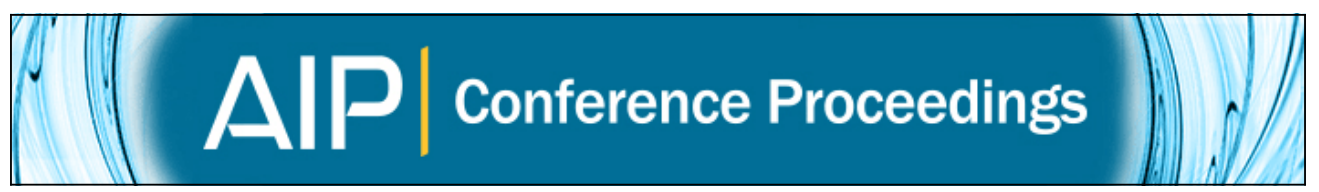

\title{
A Preliminary Analysis of a New Chandra Observation (ObsID 6148) of
}

\section{Cir X-1.}

R. Iaria, A. D'Aí, T. Di Salvo, G. Lavagetto, L. Burderi, and N. R. Robba

Citation: AIP Conference Proceedings 968, 248 (2008); doi: 10.1063/1.2840408

View online: http://dx.doi.org/10.1063/1.2840408

View Table of Contents: http://scitation.aip.org/content/aip/proceeding/aipcp/968?ver=pdfcov

Published by the AIP Publishing

Articles you may be interested in

The X-ray behaviour of Cygnus X-3

AIP Conf. Proc. 1054, 13 (2008); 10.1063/1.3002497

The complex enviroment around Cir X-1

AIP Conf. Proc. 968, 245 (2008); 10.1063/1.2840407

Power spectra of black holes $(\mathrm{BH})$ and neutron stars (NS) as a probe of hydrodynamical structure of the source: Diffusion theory and its application to X-ray observations of NS and $\mathrm{BH}$ sources

AIP Conf. Proc. 910, 334 (2007); 10.1063/1.2752484

X-ray observations of low-mass X-ray binaries: Accretion instabilities on long and short timescales

AIP Conf. Proc. 575, 261 (2001); 10.1063/1.1387317

RXTE observation of Cygnus X-1: spectra and timing

AIP Conf. Proc. 410, 849 (1997); 10.1063/1.53987 


\title{
A Preliminary Analysis of a New Chandra Observation (ObsID 6148) of Cir X-1.
}

\author{
R. Iaria*, A. D’Aí, T. Di Salvo*, G. Lavagetto*, L. Burderi ${ }^{\dagger}$ and N. R. \\ Robba* \\ ${ }^{*}$ Dipartimento di Scienze Fisiche ed Astronomiche, Università di Palermo, via Archirafi n.36, \\ 90123 Palermo, Italy. \\ ${ }^{\dagger}$ Dipartimento di Fisica, Università degli studi di Cagliari, Cittadella Universitaria, S.P. \\ Monserrato, Sestu km 0,700 09042, Monserrato (Ca), Italy.
}

\begin{abstract}
We present the preliminary spectral analysis of a $25 \mathrm{ks}$ long Chandra observation of the peculiar source $\mathrm{Cir} \mathrm{X}-1$ near the periastron passage. We estimate more precise coordinates of the source compatible with the optical and radio counterpart coordinates. We detect emission lines associated to Mg XII, Si XIII, Si XIV, S Xv, S Xvi Ar XVII, Ar XvIII, Ca XIX, Ca Xx, Fe XXv, Fe XXVI showing a redshift of $470 \mathrm{~km} \mathrm{~s}^{-1}$. The more intense emission features at $6.6 \mathrm{keV}$ show a double-peaked shape that can be modelled with two or three Gaussian lines.
\end{abstract}

Keywords: accretion discs - stars: individual: Cir X-1 — stars: neutron stars - X-ray: stars $\mathrm{X}$-ray: spectrum - X-ray: general

PACS: $97.80 . \mathrm{Jp}$

\section{INTRODUCTION}

Cir X-1 is a peculiar low-mass X-ray binary (LMXB) that recently showed ultrarelativistic outflows similar to those produced by active galactic nuclei [1]. Because of its rapid variability, a black hole was supposed to be the compact object in this system [2] until type I X-ray bursts were detected ([3], [4]), suggesting that the compact object is a neutron star.

The classical scenario accepted for Cir X-1 argues that the source has a companion star of $3-5 \mathrm{M}_{\odot}$ (probably a subgiant; see [5]), an orbital period of 16.6 days, deduced from the radio and X-ray light curve of the source, and an eccentric orbit with e $\simeq 0.7-0.9$ ([6]; [7]). Since the large X-ray flaring activity occurs at zero phase, it is also accepted that the phase zero corresponds to the periastron passage and the semi-major axis is almost aligned to the line of sight ([5]). The presence of a large amount of neutral matter occulting almost totally the $\mathrm{X}$-ray emitting source at the periastron has suggested that Cir X-1 could have a geometry similar to the Seyfert 2 Galaxies ([8]), where a torus of neutral matter is present in the outer region of the system; a similar scenario for a Galactic source implies that the inclination angle should be around $90^{\circ}$, that is $\mathrm{Cir} \mathrm{X}-1$ is an edge-on source. The works [9] and [10] go toward this direction; their authors, analysing two Chandra observations of Cir X-1 near the periastron passage, detected, for the first time in the X-ray band, the presence of P-Cygni profiles associated to highly ionized elements. The authors proposed that a presence of an accretion disk wind ([11]) could explain these features. An opposite scenario has recently been proposed by [1] and [12]. Their scenario is based on the radio detection of an ultrarelativistic jet coming

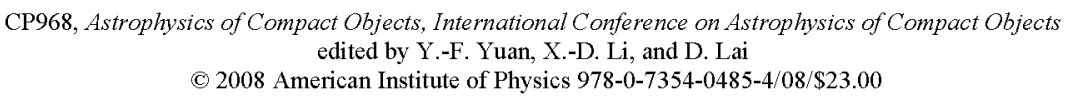


from Cir X-1 and forming an angle between its direction and the line of sight less than $5^{\circ}$. Following this scenario the jet should be almost perpendicular to the equatorial plane implying that the inclination angle is less than $5^{\circ}$ and Cir $\mathrm{X}-1$ is a face-on source.

\section{DATA ANALYSIS AND PRELIMINARY RESULTS}

Cir X-1 was observed with the Chandra observatory on 2005 Jan 03 from 01:14:52 to 08:41:32 UT using the HETGS for a total observation time of $25 \mathrm{ks}$ (OBS. ID. 6148, from the Chandra public archive). The corresponding phase was $0.974-0.993$ using the ephemeris of Stewart et al. (1993). We have obtained the following coordinates: R.A. $=15^{h} 20^{m} 40^{s} .874$, Dec. $=-57^{\circ} 10^{\prime} 00^{\prime \prime} .26(\mathrm{~J} 2000.0)$ with an uncertainty circle of the absolute position of $0.6^{\prime \prime}$.

We fit the extracted spectra in the $1.3-4 \mathrm{keV}$ and $1.3-7.7 \mathrm{keV}$ energy range for firstorder MEG and first-order HEG, respectively, using as continuum an absorbed powerlaw with photon index $1.8 \pm 0.4$ additionally absorbed by a partial covering component with an equivalent hydrogen column density of $(6.3 \pm 1.8) \times 10^{22} \mathrm{~cm}^{-2}$. The interstellar equivalent hydrogen column density was kept fixed at $1.85 \times 10^{22} \mathrm{~cm}^{-2}$, similar to the value obtained by [10]. The presence of several emission features is clearly evident in the residuals. We detect the emission lines of He-like and H-like ions: the $\mathrm{Ly}_{\alpha}$ and $\mathrm{Ly}_{\beta}$ transitions of Mg XII, Si XIV and S XVI; the $\mathrm{Ly}_{\alpha}$ transitions of Ar XvIII, Ca Xx , and Fe XXVI; the forbidden and resonance lines of Si XIII and S XV; the resonance line of Ar XVII and Ca XIX, and the intercombination line of Fe XXV (see Fig. 1). The centroids of the lines show a redshift with an average value of $\mathrm{z}$ of $(1.6 \pm 0.3) \times 10^{-3}$. The line at $6.66 \mathrm{keV}$, fitted as the Fe $\mathrm{xxV}$ intercombination line, shows a double-peaked structure (see Fig. 1). We propose two possible scenarios to well-model the complex shape of this feature. The first scenario is that we are observing the blending of the forbidden, intercombination and resonance line of the Fe $x \times v$ triplet, then we fit the feature using three Gaussian line. In this hypothesis, to disentangle the lines of the Fe Xxv triplet we keep fixed the continuum parameters, add three lines imposing a difference between their centroid energies equal to that in the rest frame, and that the line width is same for the three lines. The second scenario to explain the double peaked shape of the $6.66 \mathrm{keV}$ line is that we are observing the redshifted and blueshifted Fe $\mathbf{x x v}$ intercombination lines. To test this hypothesis we fix the continuum parameters and add two Gaussian lines to fit the excess at $6.66 \mathrm{keV}$. We will discuss the physical implications of these results in our work in preparation.

\section{REFERENCES}

1. R. Fender, K. Wu, H. Johnston, T. Tzioumis, P. Jonker, R. Spencer, and M. van der Klis, Nature 427, 222-224 (2004), arXiv : astro-ph/ 0401290.

2. A. Toor, ApJ 215, L57-L60 (1977).

3. A. F. Tennant, A. C. Fabian, and R. A. Shafer, MNRAS 221, 27P-31P (1986).

4. A. F. Tennant, A. C. Fabian, and R. A. Shafer, MNRAS 219, 871-881 (1986).

5. H. M. Johnston, R. Fender, and K. Wu, MNRAS 308, 415-423 (1999), arXiv: astro-ph/ 9904112 . 

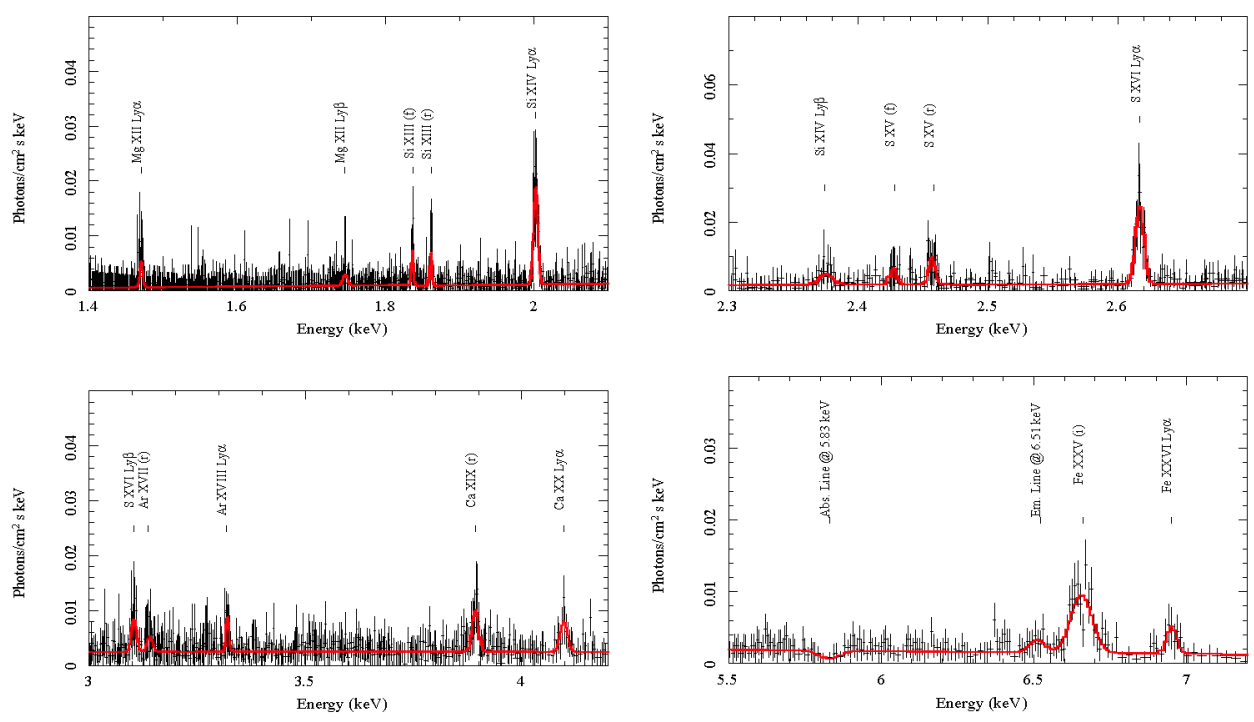

FIGURE 1. The panels show the detected continuum emission and the emission lines in the energy range $1.4-7.7 \mathrm{keV}$. An absorption features at $5.83 \mathrm{keV}$ is also observed with a $2.3 \sigma$ level significance. The emission feature at $6.6 \mathrm{keV}$ is here fitted using only one Gaussian line.
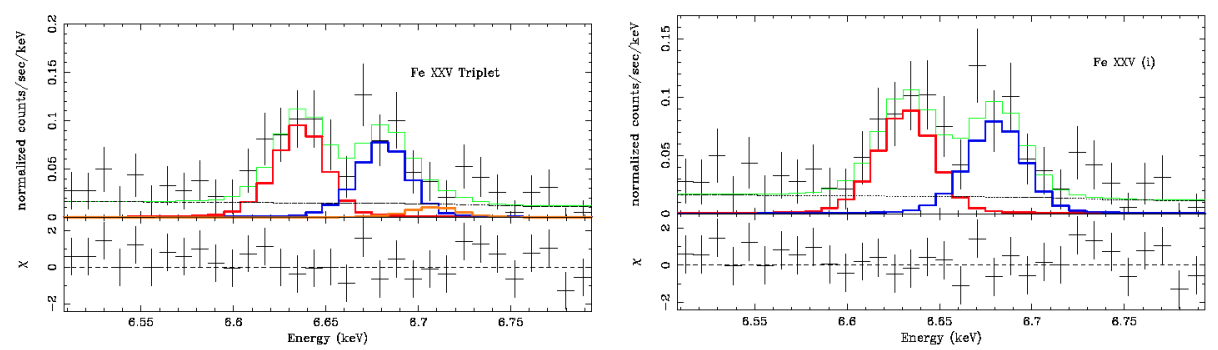

FIGURE 2. The emission feature at $6.6 \mathrm{keV}$ can be fitted either with the three lines of the Fe XXV triplet with a very low flux associated to the resonance line (left panel) or with the red and blue-shifted components associated to the intercombination line of Fe XXV (right panel).

6. P. Murdin, D. L. Jauncey, I. Lerche, G. D. Nicolson, L. J. Kaluzienski, S. S. Holt, and R. F. Haynes, $A \& A$ 87, 292-298(1980).

7. T. M. Tauris, R. P. Fender, E. P. J. van den Heuvel, H. M. Johnston, and K. Wu, MNRAS 310, 1165-1169 (1999), arXiv: astro-ph/9909148.

8. W. N. Brandt, A. C. Fabian, T. Dotani, F. Nagase, H. Inoue, T. Kotani, and Y. Segawa, MNRAS 283, 1071-1082 (1996), arXiv:astro-ph/9607018.

9. W. N. Brandt, and N. S. Schulz, ApJ 544, L123-L127 (2000), arXiv : astro-ph/ 0007406.

10. N. S. Schulz, and W. N. Brandt, $A p J$ 572,971-983 (2002), arXiv : astro-ph/0112483.

11. M. C. Begelman, C. F. McKee, and G. A. Shields, ApJ 271, 70-88 (1983).

12. V. Tudose, R. P. Fender, C. R. Kaiser, A. K. Tzioumis, M. van der Klis, and R. E. Spencer, MNRAS 372, 417-424 (2006), arXiv: astro-ph/0507612. 